\title{
1,2-Sulfanyl Group Migration as Driving Force: New Approach to Pyrroles by Reaction of Allenic aldehydes with Amines
}

\author{
Lingling Peng, Xiu Zhang, Jie Ma, Zhenzhen Zhong and Jianbo Wang*
}

Beijing National Laboratory of Molecular Sciences (BNLMS), Green Chemistry Center (GCC) and Key Laboratory of Bioorganic Chemistry and Molecular Engineering of Ministry of Education, College of Chemistry, Peking University, Beijing 100871, China

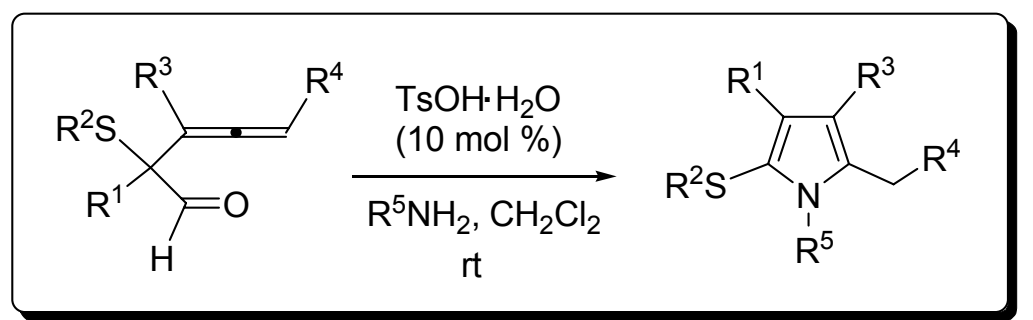

Contents

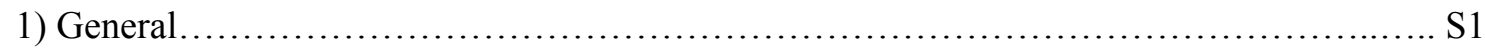

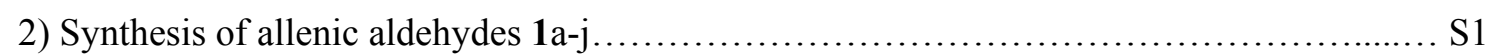

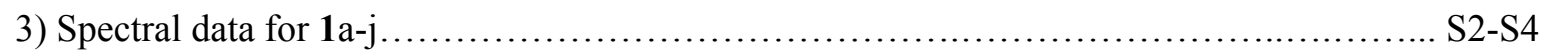

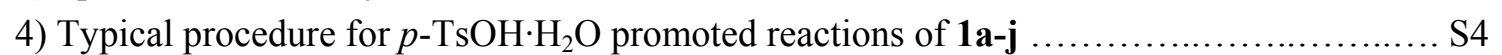

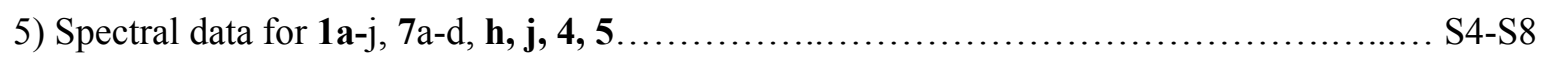




\section{Experimental Section}

Caution: All diazo compounds are highly toxic or presumed to be toxic. Diazo compounds are potentially explosive. They should be handled with care in a well-ventilated fumehood.

General All solvents were distilled prior to use. $\mathrm{CH}_{2} \mathrm{Cl}_{2}$ and $\mathrm{ClCH}_{2} \mathrm{CH}_{2} \mathrm{Cl}$ was distilled over NaH. Toluene was distilled over Na. For chromatography, 200-300 mesh silica gel (Yantai, China) was employed. ${ }^{1} \mathrm{H}$ and ${ }^{13} \mathrm{C}$ NMR spectra were recorded at $300 \mathrm{MHz}$ and $75 \mathrm{MHz}$ with Varian Mercury 300 spectrometer. Chemical shifts are reported in ppm using tetramethylsilane as internal standard. IR spectra were recorded with a Nicolet 5MX-S infrared spectrometer. Mass spectra were obtained on a VG ZAB-HS mass spectrometer. Elemental analysis was measured with Elementar Vario apparatus.

\section{The Synthesis of allenic aldehydes 1a-j:}

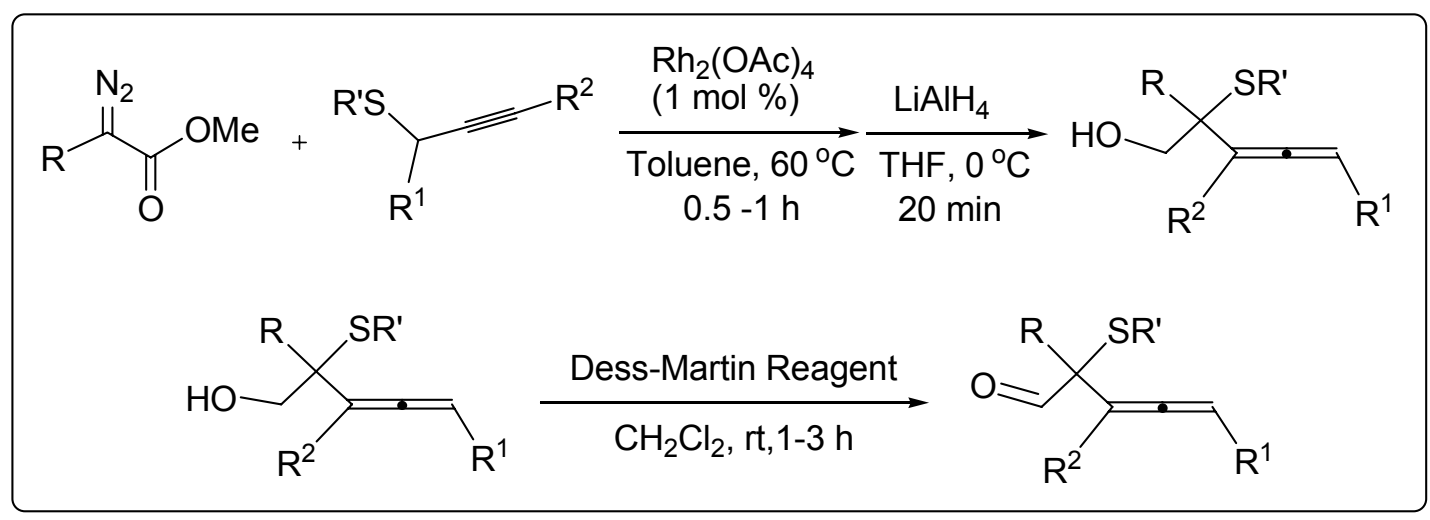

\section{Experimental procedures}

Under a nitrogen atmosphere, $\mathrm{Rh}_{2}(\mathrm{OAc})_{4}(0.020 \mathrm{mmol})$ and propargyl sulfide $(2.0 \mathrm{mmol})$ were mixed in dry toluene $(15 \mathrm{~mL})$ in a $100 \mathrm{~mL}$ round-bottomed flask. To the solution was then adding dropwisely diazoacetate $(2.0 \mathrm{mmol})$ in anhydrous toluene $(15 \mathrm{~mL})$ at $60{ }^{\circ} \mathrm{C}$ in about 20 minutes. The reaction was kept at the same temperature until the yellow solution turned light green or colorless and the diazoacetate compound disappeared as judged by TLC. Then $10 \mathrm{~mL}$ anhydrous THF was added to the mixture, and $\mathrm{LiAlH}_{4}(5.0 \mathrm{mmol})$ was added by portion at $0{ }^{\circ} \mathrm{C}$. The mixture was stirred for another $20 \mathrm{~min}$ at the same temperature. Then $5 \mathrm{~mL}$ EtOAc was added and stirred for $20 \mathrm{~min}$ at $0{ }^{\circ} \mathrm{C}$. At last $10 \mathrm{~mL} 1 \mathrm{~N} \mathrm{HCl}$ was added. After removal of the organic solvent in vacuum the residue was extracted with $\mathrm{CH} 2 \mathrm{Cl} 2$. The combined organic layers were dried over $\mathrm{Na} 2 \mathrm{SO} 4$ and evaporated, and the residue was purified by silica gel column eluted with petroleum ether/ EtOAc (20:1) to afford the corresponding allenic alcohol.

A solution of allenic alcohol $(1.0 \mathrm{mmol})$ in $\mathrm{CH}_{2} \mathrm{Cl}_{2}(15 \mathrm{~mL})$ was cooled to $0{ }^{\circ} \mathrm{C}$. Then Dess-Martin Reagent $(1.2 \mathrm{mmol})$ was added by portion. The mixture was stirred at room temperature for another 1-3 hours. $15 \mathrm{ml} 5 \% \mathrm{NaOH}$ was added after the reaction was completed. The mixture was extracted with $\mathrm{CH} 2 \mathrm{Cl} 2$ and the combined organic layers were dried over $\mathrm{Na} 2 \mathrm{SO} 4$. After removal of organic solvent in vacuum the residue was purified by silica gel column eluted with petroleum ether/ EtOAc $(200: 1)$ to afford the corresponding allenic aldehyde. 


\section{2-Phenyl-2-(phenylthio)penta-3,4-dienal (1a)}

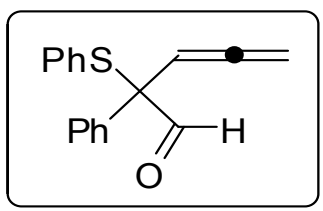

White solid; mp: $65-66{ }^{\circ} \mathrm{C}$; IR (film) 1951, 1719, 854, 749, $693 \mathrm{~cm}^{-1}$; ${ }^{1} \mathrm{H}$ NMR $\left(\mathrm{CDCl}_{3}, 300 \mathrm{MHz}\right) \delta 4.61(\mathrm{dd}, J=6.6,11.7 \mathrm{~Hz}, 1 \mathrm{H}), 4.79(\mathrm{dd}, J=6.6,11.7 \mathrm{~Hz}$, $1 \mathrm{H}), 5.54(\mathrm{t}, J=6.6 \mathrm{~Hz}, 1 \mathrm{H}), 7.25-7.49(\mathrm{~m}, 10 \mathrm{H}), 9.52(\mathrm{~s}, 1 \mathrm{H}) ;{ }^{13} \mathrm{C}$ NMR $\left(\mathrm{CDCl}_{3}, 75 \mathrm{MHz}\right) \delta 65.76,78.36,90.57,128.42,128.60,128.81,129.56$, 129.67, 135.62, 137.29, 190.26, 209.19. EI-MS ( $\mathrm{m} / \mathrm{z}$, relative intensity): 266 (M+1, 8), 237 (12), 223 (5), 157 (75), 128 (100), 109 (26). Anal. calcd for $\mathrm{C}_{17} \mathrm{H}_{14} \mathrm{OS}$ : C, 76.66; H, 5.30. Found: C, 76.75; H, 5.30.

\section{2-(4-Cholorophenyl)-2-(phenylthio)penta-3,4-dienal (1b)}

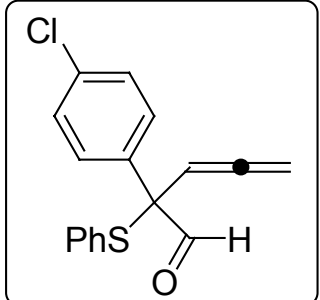

4.36 .

Oil; IR (film) 1951, 1718, 1491, 1095, 1014, 854, 831, 750, $691 \mathrm{~cm}^{-1} ;{ }^{1} \mathrm{H}$ NMR $\left(\mathrm{CDCl}_{3}, 300 \mathrm{MHz}\right) \delta 4.67(\mathrm{dd}, J=6.6,12.0 \mathrm{~Hz}, 1 \mathrm{H}), 4.82(\mathrm{dd}, J=6.6$, $12.0 \mathrm{~Hz}, 1 \mathrm{H}), 5.50(\mathrm{t}, J=6.6 \mathrm{~Hz}, 1 \mathrm{H}), \quad 7.27-7.43(\mathrm{~m}, 9 \mathrm{H}), 9.48(\mathrm{~s}, 1 \mathrm{H}),{ }^{13} \mathrm{C}$ $\mathrm{NMR}\left(\mathrm{CDCl}_{3}, 75 \mathrm{MHz}\right) \delta 64.98,78.82,90.36,128.67,128.90,129.10,129.85$, 129.91, 134.19, 134.36, 137.23, 189.82, 209.04. EI-MS $(\mathrm{m} / z$, relative intensity): $300\left(\mathrm{M}^{+}, 13\right), 271$ (11), 236 (7), 191 (83), 163 (21), 128 (100), 109 (25). Anal. calcd for $\mathrm{C}_{17} \mathrm{H}_{13}{ }^{35} \mathrm{ClOS}$ : $\mathrm{C}, 67.93 ; \mathrm{H}, 4.35$. Found: $\mathrm{C}, 67.88 ; \mathrm{H}$,

\section{2-(3,4-Dicholorophenyl)-2-(phenylthio)penta-3,4-dienal (1c)}

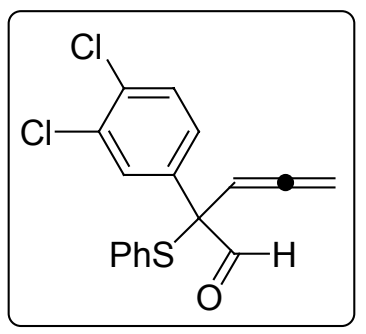

[M] 333.9986. Found: 333.9985.

Oil; IR (film) 1951, 1721, 1472, 1139, 1030, 856, 749, $691 \mathrm{~cm}^{-1} ;{ }^{1} \mathrm{H}$ NMR $\left(\mathrm{CDCl}_{3}, 300 \mathrm{MHz}\right) \delta 4.74(\mathrm{dd}, J=6.6,12.0 \mathrm{~Hz}, 1 \mathrm{H}), 4.88(\mathrm{dd}, J=6.6,12.0$ $\mathrm{Hz}, 1 \mathrm{H}), 5.49(\mathrm{t}, J=6.6 \mathrm{~Hz}, 1 \mathrm{H}), 7.27-7.46(\mathrm{~m}, 7 \mathrm{H}), 7.55-7.56(\mathrm{~m}, 1 \mathrm{H})$, $9.47(\mathrm{~s}, 1 \mathrm{H}) ;{ }^{13} \mathrm{C} \mathrm{NMR}\left(\mathrm{CDCl}_{3}, 75 \mathrm{MHz}\right) \delta 64,37,79.27,90.05,128.00$, $128.81,129.01,130.06,130.28,130.69,132.59,132.65,136.05,137.23$, 189.46, 209.06. EI-MS ( $\mathrm{m} / \mathrm{z}$, relative intensity): $334\left(\mathrm{M}^{+}, 13\right), 305$ (10), 270 (7), 225 (71), 162 (100), 126 (31), 109 (39). HRMS calcd for $\mathrm{C}_{17} \mathrm{H}_{12}{ }^{35} \mathrm{Cl}_{2} \mathrm{OS}$

\section{2-(4-Methoxyphenyl)-2-(phenylthio)penta-3,4-dienal (1d)}

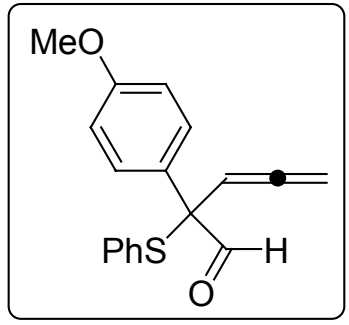

White solid; mp: $56-58{ }^{\circ} \mathrm{C}$; IR (film) 2837, 1952, 1716, 1509, 1253, 1182, 1034, 831, 750, $692 \mathrm{~cm}^{-1} ;{ }^{1} \mathrm{H}$ NMR $\left(\mathrm{CDCl}_{3}, 300 \mathrm{MHz}\right) \delta 3.81(\mathrm{~s}, 3 \mathrm{H}), 4.62$ (dd, $J=6.6,11.7 \mathrm{~Hz}, 1 \mathrm{H}), 4.79(\mathrm{dd}, J=6.6,11.7 \mathrm{~Hz}, 1 \mathrm{H}), 5.51(\mathrm{t}, J=6.6 \mathrm{~Hz}$, $1 \mathrm{H}), 6.89-76.94(\mathrm{~m}, 2 \mathrm{H}), 7.27-7.45(\mathrm{~m}, 7 \mathrm{H}), 9.48(\mathrm{~s}, 1 \mathrm{H}) ;{ }^{13} \mathrm{C} \mathrm{NMR}\left(\mathrm{CDCl}_{3}\right.$, $75 \mathrm{MHz}) \delta 55.21,65.29,78.32,90.68,113.94,127.32,128.78,129.61$, 129.71, 137.25, 159.47, 190.44, 209.11. EI-MS ( $\mathrm{m} / \mathrm{z}$, relative intensity): 296 $\left(\mathrm{M}^{+}, 5\right), 267$ (4), 234 (3), 187 (100), 159 (40), 144 (28), 128 (28), 115 (66), 109 (12). Anal. calcd for $\mathrm{C}_{18} \mathrm{H}_{16} \mathrm{O}_{2} \mathrm{~S}: \mathrm{C}, 72.94 ; \mathrm{H}, 5.44$. Found: $\mathrm{C}, 72.88 ; \mathrm{H}, 5.40$.

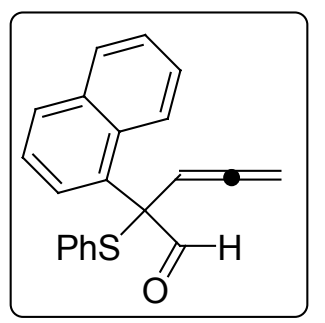

\section{2-(1-Naphthyl)-2-(phenylthio)penta-3,4-dienal (1e)}

Oil; IR (film) 1952, 1702, 850, 801, 776, 751, $691 \mathrm{~cm}^{-1} ;{ }^{1} \mathrm{H}$ NMR $\left(\mathrm{CDCl}_{3}, 300\right.$ MHz) $\delta 4.29(\mathrm{dd}, J=6.6,12.0 \mathrm{~Hz}, 1 \mathrm{H}), 4.61(\mathrm{dd}, J=6.6,12.0 \mathrm{~Hz}, 1 \mathrm{H}), 5.69$ (t, $J=6.6 \mathrm{~Hz}, 1 \mathrm{H}), 7.32-7.54(\mathrm{~m}, 8 \mathrm{H}), 7.86-7.94(\mathrm{~m}, 3 \mathrm{H}), 7.98-8.01(\mathrm{~m}, 1 \mathrm{H}), 9.27$ $(\mathrm{s}, 1 \mathrm{H}) ;{ }^{13} \mathrm{C} \mathrm{NMR}\left(\mathrm{CDCl}_{3}, 75 \mathrm{MHz}\right) \delta 66.90,77.82,90.68,124.93,125.16$, 
$125.65,126.39,126.96,128.61,128.85,129.00,129.85,130.02,131.20,131.40,134.21,138.23$, 187.08, 209.20. EI-MS ( $m / z$, relative intensity): $316\left(\mathrm{M}^{+}, 14\right), 287$ (7), 254 (3), 207 (94), 179 (100), 152 (25), 109 (11). HRMS calcd for $\mathrm{C}_{21} \mathrm{H}_{16} \mathrm{OS}$ [M] 316.0922. Found: 316.0925.

\section{2-(4-Bromophenyl)-2-(phenylthio)penta-3,4-dienal (1f)}

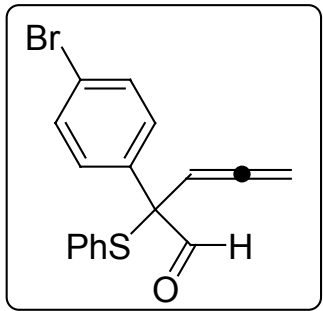

Oil; IR (film) 1951, 1718, 1486, 1076, 1009, 855, 750, $691 \mathrm{~cm}^{-1} ;{ }^{1} \mathrm{H}$ NMR $\left(\mathrm{CDCl}_{3}, 300 \mathrm{MHz}\right) \delta 4.67(\mathrm{dd}, J=6.6,11.7 \mathrm{~Hz}, 1 \mathrm{H}), 4.82(\mathrm{dd}, J=6.6,11.7 \mathrm{~Hz}$, $1 \mathrm{H}), 5.49(\mathrm{t}, J=6.6 \mathrm{~Hz}, 1 \mathrm{H}), 7.27-7.42(\mathrm{~m}, 7 \mathrm{H}), 7.49-7.53(\mathrm{~m}, 2 \mathrm{H}), 9.48(\mathrm{~s}$, $1 \mathrm{H}) ;{ }^{13} \mathrm{C}$ NMR $\left(\mathrm{CDCl}_{3}, 75 \mathrm{MHz}\right) \delta 65.04,78.85,90.29,122.63,128.91$, $129.10,129.85,130.21,131.63,134.75,137.23,189.75,209.05$. EI-MS $(m / z$, relative intensity): $344\left(\mathrm{M}^{+}, 9\right), 315$ (6), 235 (68), 156 (39), 128 (100), 109 (34). HRMS calcd for $\mathrm{C}_{17} \mathrm{H}_{13} \mathrm{OS}^{79} \mathrm{Br}\left[\mathrm{M}^{+}\right]$343.9871. Found: 343.9874 .

\section{2- Phenylthio-2-phenylpenta-3,4-dienal (1g)}

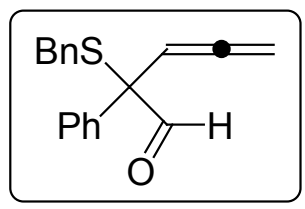

Oil; IR (film) 1951, 1709, 1495, 853, $697 \mathrm{~cm}^{-1} ;{ }^{1} \mathrm{H} \mathrm{NMR}\left(\mathrm{CDCl}_{3}, 300 \mathrm{MHz}\right) \delta$ $3.56(\mathrm{~d}, J=11.7 \mathrm{~Hz}, 1 \mathrm{H}), 3.66(\mathrm{~d}, J=11.7 \mathrm{~Hz}, 1 \mathrm{H}), 4.83-4.95(\mathrm{~m}, 2 \mathrm{H}), 5.74(\mathrm{t}$, $J=6.6 \mathrm{~Hz}, 1 \mathrm{H}), 7.21-7.41(\mathrm{~m}, 8 \mathrm{H}), 7.48-7.51(\mathrm{~m}, 2 \mathrm{H}), 9.38(\mathrm{~s}, 1 \mathrm{H}) ;{ }^{13} \mathrm{C} \mathrm{NMR}$ $\left(\mathrm{CDCl}_{3}, 75 \mathrm{MHz}\right) \delta 34.56,63.08,78.83,90.67,127.35,128.13,128.29,128.56$, $128.71,129.19,135.92,136.00,188.43,209.18$. EI-MS ( $\mathrm{m} / \mathrm{z}$, relative intensity): $280\left(\mathrm{M}^{+}, 1\right), 251$ (5), 217 (1), 189 (18), 157 (30), 128 (40), 91 (100). HRMS calcd for $\mathrm{C}_{18} \mathrm{H}_{16} \mathrm{OS}$ [M] 280.0927. Found: 280.0927.

\section{3-Methyl-2-phenyl-2-(phenylthio)penta-3,4-dienal (1h)}

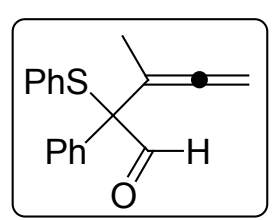

Oil; IR (film) 1951, 1720, 1439, 858, 748, $693 \mathrm{~cm}^{-1} ;{ }^{1} \mathrm{H}$ NMR $\left(\mathrm{CDCl}_{3}, 300 \mathrm{MHz}\right)$ $\delta 1.67(\mathrm{t}, J=3.0 \mathrm{~Hz}, 3 \mathrm{H}), 4.59-4.66(\mathrm{~m}, 1 \mathrm{H}), 4.80-4.87(\mathrm{~m}, 1 \mathrm{H}), 7.20-7.37(\mathrm{~m}$, $8 \mathrm{H}), 7.41-7.45(\mathrm{~m}, 2 \mathrm{H}), 9.47(\mathrm{~s}, 1 \mathrm{H}) ;{ }^{13} \mathrm{C} \mathrm{NMR}\left(\mathrm{CDCl}_{3}, 75 \mathrm{MHz}\right) \delta 16.60,68.76$, $77.77,96.41,127.95,128.27,128.55,129.10,129.23,130.07,134.97,136.40$, 191.52, 207.82. EI-MS ( $\mathrm{m} / \mathrm{z}$, relative intensity): $280\left(\mathrm{M}^{+}, 1\right), 265$ (1), 251 (2), 218 (2), 171 (100), 143 (29), 128 (72), 115 (31). HRMS calcd for $\mathrm{C}_{18} \mathrm{H}_{16} \mathrm{OS}$ [M] 280.0922. Found: 280.0920 .

\section{5-Butyl-2-phenyl-2-(phenylthio)penta-3,4-dienal (1i)}

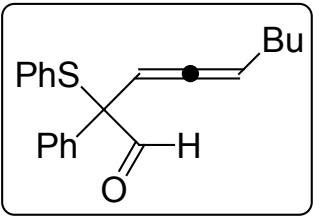

Oil; IR (film) 2956, 2928, 1961, 1717, 1582, 1439, 872, 749, $693 \mathrm{~cm}^{-1} ;{ }^{1} \mathrm{H}$ $\operatorname{NMR}\left(\mathrm{CDCl}_{3}, 300 \mathrm{MHz}\right) \delta 0.80(\mathrm{t}, J=7.2 \mathrm{~Hz}, 3 \mathrm{H}), 0.87(\mathrm{t}, J=7.2 \mathrm{~Hz}, 3 \mathrm{H})$, $1.02-1.19(\mathrm{~m}, 4 \mathrm{H}), 1.20-1.34(\mathrm{~m}, 4 \mathrm{H}), 1.64-1.78(\mathrm{~m}, 2 \mathrm{H}), 1.88-1.96(\mathrm{~m}, 2 \mathrm{H})$, $5.04(\mathrm{dd}, J=6.9,13.2 \mathrm{~Hz}, 1 \mathrm{H}), 5.23(\mathrm{dd}, J=6.9,13.2 \mathrm{~Hz}, 1 \mathrm{H}), 5.45-5.52(\mathrm{~m}$, $2 \mathrm{H}), 7.24-7.48(\mathrm{~m}, 2 \mathrm{H}), 9.53(\mathrm{~s}, 2 \mathrm{H}) ;{ }^{13} \mathrm{C} \mathrm{NMR}\left(\mathrm{CDCl}_{3}, 75 \mathrm{MHz}\right) \delta 13.77$, $13.82,21.98,22.04,27.80,27.87,30.88,30.96,65.92,66.11,91.18,91.31,94.97,95.20,128.22$, $128.41,128.54,128.56,128.70,128.75,129.43,129.51,129.80,129.87,135.97,136.07,137.06$, 137.20, 190.83, 190.93, 205.10, 205.15. EI-MS ( $\mathrm{m} / z$, relative intensity): $322\left(\mathrm{M}^{+}, 9\right), 293$ (4), 265 (26), 213 (36), 157 (100), 129 (56), 115 (50), 109 (36), 91 (27). HRMS calcd for $\mathrm{C}_{21} \mathrm{H}_{22} \mathrm{OS}$ [M] 322.1391. Found: 322.1398 . 


\section{2-Methyl-2-(phenylthio)penta-3,4-dienal (1j)}

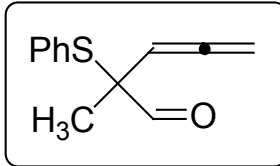

Oil; IR (film) 3062, 2815, 1952, 1717, 1474, 1439, 1069, 857, 751, $693 \mathrm{~cm}^{-1} ;{ }^{1} \mathrm{H}$ NMR $\left(\mathrm{CDCl}_{3}, 300 \mathrm{MHz}\right) \delta 1.40(\mathrm{~s}, 3 \mathrm{H}), 4.89-5.01(\mathrm{~m}, 2 \mathrm{H}), 5.32(\mathrm{t}, J=6.6 \mathrm{~Hz}$, $1 \mathrm{H}), 7.27-7.45(\mathrm{~m}, 5 \mathrm{H}), 9.38(\mathrm{~s}, 1 \mathrm{H}) ;{ }^{13} \mathrm{C} \mathrm{NMR}\left(\mathrm{CDCl}_{3}, 75 \mathrm{MHz}\right) \delta$ 18.97, 57.09, $79.00,90.09,128.92,129.32,129.76,137.21,192.54,208.16$. EI-MS $(\mathrm{m} / \mathrm{z}$, relative intensity): $204\left(\mathrm{M}^{+}, 6\right), 175$ (41), 160 (12), 147 (13), 110 (41), 95 (100). HRMS calcd for $\mathrm{C}_{12} \mathrm{H}_{12} \mathrm{OS}[\mathrm{M}]$ 204.0609. Found: 204.0616.

\section{Typical Procedure for $\boldsymbol{p}$-TsOH$\cdot \mathrm{H}_{2} \mathrm{O}$ Promoted Reactions of 1a-j:}

Aniline $(1.5 \mathrm{mmol})$, allenic aldehyde $(1.0 \mathrm{mmol})$ and $p-\mathrm{TsOH} \cdot \mathrm{H}_{2} \mathrm{O}(0.1 \mathrm{mmol})$ were mixed in dry $\mathrm{CH}_{2} \mathrm{Cl}_{2}$, the system was stirred at the room temperature until the reaction was completed as judged by TLC. Removal of the solvent in vacuo to give a crude residue which was purified by silica gel with petroleum ether/ethyl acetate $(200: 1)$ to afford pure product .

\section{5-Methyl-1,3-diphenyl-2-(phenylthio)pyrrole (2a)}

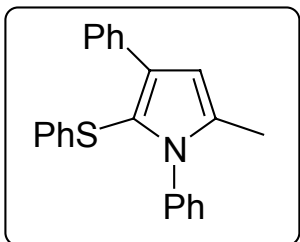

White solid; mp: $81{ }^{\circ} \mathrm{C}$; IR (film) 3062, 1598, 1510, 1497, 1477, 1398, 1358, $761,739,695 \mathrm{~cm}^{-1} ;{ }^{1} \mathrm{H}$ NMR $\left(\mathrm{CDCl}_{3}, 300 \mathrm{MHz}\right) \delta 2.10(\mathrm{~s}, 3 \mathrm{H}), 6.42(\mathrm{~s}, 1 \mathrm{H})$, 6.86-6.89 (m, 2H), 6.99-7.14 (m, 5H), 7.18-7.35 (m, 6H), 7.67-7.70 (m, 2H); ${ }^{13} \mathrm{C} \mathrm{NMR}\left(\mathrm{CDCl}_{3}, 75 \mathrm{MHz}\right) \delta 13.58,108.39,114.08,124.85,125.62,126.28$, $127.85,128.08,128.16,128.49,128.60,128.62,132.59,134.16,135.51$, 137.95,139.64. EI-MS ( $m / z$, relative intensity): $341\left(\mathrm{M}^{+}, 100\right), 308$ (13), 264 (14), 249 (10), 118 (13), 77 (29). HRMS calcd for $\mathrm{C}_{23} \mathrm{H}_{19} \mathrm{NS}$ [M] 341.1238. Found: 341.1235.

\section{1-(4-Methylphenyl)-5-methyl-3-phenyl-2-(phenylthio)pyrrole (7a)}

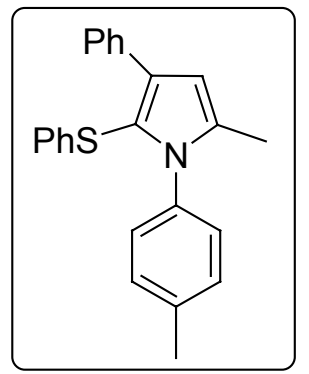

White solid; mp: $79-81{ }^{\circ} \mathrm{C}$; IR (film) 2917, 1582, 1513, 828, 761, 737, $694 \mathrm{~cm}^{-1}$; ${ }^{1} \mathrm{H}$ NMR $\left(\mathrm{CDCl}_{3}, 300 \mathrm{MHz}\right) \delta 2.10(\mathrm{~s}, 3 \mathrm{H}), 2.33(\mathrm{~s}, 3 \mathrm{H}), 6.41(\mathrm{~s}, 1 \mathrm{H}), 6.88-7.34$ (m, 12H), 7.66-7.68 (m, 2H); ${ }^{13} \mathrm{C}$ NMR $\left(\mathrm{CDCl}_{3}, 75 \mathrm{MHz}\right) \delta 13.59,21.14,108.26$, $113.95,124.76,125.43,126.22,127.80,128.14,128.22,128.61,129.18,132.45$, 134.28, 135.34, 135.56, 137.94, 139.90. EI-MS (m/z, relative intensity): 355 $\left(\mathrm{M}^{+}, 100\right), 322$ (6), 278 (10), 263 (7), 128 (7), 91 (12). HRMS calcd for $\mathrm{C}_{24} \mathrm{H}_{21} \mathrm{NS}$ [M] 355.1395. Found: 355.1398 .

\section{1-(4-Bromophenyl)-5-methyl-3-phenyl-2-(phenylthio)pyrrole (7b)}

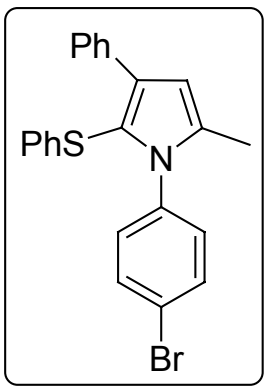

White solid; mp: 103-104 ${ }^{\circ} \mathrm{C}$; IR (film) 3059, 1490, 1070, 1007, 835, 762, 737, $694 \mathrm{~cm}^{-1} ;{ }^{1} \mathrm{H}$ NMR $\left(\mathrm{CDCl}_{3}, 300 \mathrm{MHz}\right) \delta 2.10(\mathrm{~s}, 3 \mathrm{H}), 6.42(\mathrm{~s}, 1 \mathrm{H}), 6.85-6.94(\mathrm{~m}$, $4 \mathrm{H}), 7.01-7.45(\mathrm{~m}, 8 \mathrm{H}), 7.65-7.68(\mathrm{~m}, 2 \mathrm{H}) ;{ }^{13} \mathrm{C} \mathrm{NMR}\left(\mathrm{CDCl}_{3}, 75 \mathrm{MHz}\right) \delta 13.56$, $108.79,114.09$, 122.11, 125.06, 125.61, 126.44, 127.85, 128.21, 128.76, 130.21, $131.75,132.99,134.02,135.28,136.98,139.32$. EI-MS (m/z, relative intensity): $419\left(\mathrm{M}^{+}, 100\right), 386$ (4), 342 (9), 262 (14), 128 (16), 77 (16). HRMS calcd for $\mathrm{C}_{23} \mathrm{H}_{18} \mathrm{NS}^{79} \mathrm{Br}[\mathrm{M}]$ 419.0343. Found: 419.0344 . 


\section{1-(4-Chlorophenyl)-5-methyl-3-phenyl-2-(phenylthio)pyrrole (7c)}

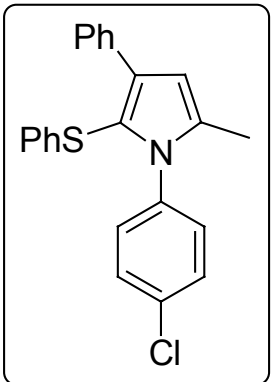

White solid; mp: $89-90{ }^{\circ} \mathrm{C}$; IR (film) 3058, 1493, 1089, 838, 762, 738, $694 \mathrm{~cm}^{-1}$; ${ }^{1} \mathrm{H}$ NMR $\left(\mathrm{CDCl}_{3}, 300 \mathrm{MHz}\right) \delta 2.10(\mathrm{~s}, 3 \mathrm{H}), 6.42(\mathrm{~s}, 1 \mathrm{H}), 6.86-7.35(\mathrm{~m}, 12 \mathrm{H})$, 7.65-7.68 (m, 2H); ${ }^{13} \mathrm{C}$ NMR $\left(\mathrm{CDCl}_{3}, 75 \mathrm{MHz}\right) \delta 13.55,108.70,114.04,125.02$, $125.54,126.42,127.81,128.19,128.74,129.83,132.91,133.97,134.06,135.25$, 136.40, 139.30. EI-MS (m/z, relative intensity): $375\left(\mathrm{M}^{+}, 100\right), 342$ (5), 298 (12), 262 (8), 128 (20), 77 (23). HRMS calcd for $\mathrm{C}_{23} \mathrm{H}_{18} \mathrm{NS}^{35} \mathrm{Cl}[\mathrm{M}]$ 375.0848. Found: 375.0845 .

\section{1-(3-Methylphenyl)-5-methyl-3-phenyl-2-(phenylthio)pyrrole (7d)}

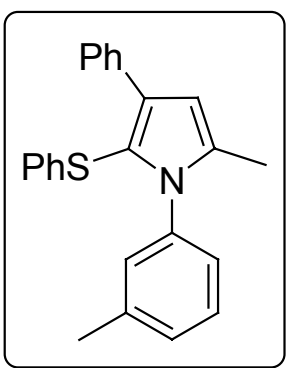

White solid; mp: $65-66{ }^{\circ} \mathrm{C}$; IR (film) 3059, 2926, 795, 762, 739, $696 \mathrm{~cm}^{-1} ;{ }^{1} \mathrm{H}$ NMR $\left(\mathrm{CDCl}_{3}, 300 \mathrm{MHz}\right) \delta 2.10(\mathrm{~s}, 3 \mathrm{H}), 2.21(\mathrm{~s}, 3 \mathrm{H}), 6.41(\mathrm{~s}, 1 \mathrm{H}), 6.80-7.34(\mathrm{~m}$, $12 \mathrm{H}), 7.68-7.70(\mathrm{~m}, 2 \mathrm{H}) ;{ }^{13} \mathrm{C} \mathrm{NMR}\left(\mathrm{CDCl}_{3}, 75 \mathrm{MHz}\right) \delta 13.57,21.11,108.21$, $114.08,124.84,125.43,125.76,126.22,127.80,128.14,128.21,128.57,128.81$, $129.32,132.47,134.08,135.55,137.82,138.36,139.80$. EI-MS (m/z, relative intensity): 355 ( $\left.\mathrm{M}^{+}, 100\right), 322$ (6), 278 (12), 263 (9), 128 (15), 91 (21), 77 (21). HRMS calcd for $\mathrm{C}_{24} \mathrm{H}_{21} \mathrm{NS}$ [M] 355.1395. Found: 355.1388.

\section{1-Benzyl-5-methyl-3-phenyl-2-(phenylthio)pyrrole (7h)}

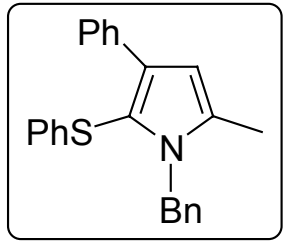

White solid; mp: $120{ }^{\circ} \mathrm{C}$; IR (film) 3061, 1511, 1477, 1341, 909, 761, 736, 693 $\mathrm{cm}^{-1}$; ${ }^{1} \mathrm{H}$ NMR $\left(\mathrm{CDCl}_{3}, 300 \mathrm{MHz}\right) \delta 2.19(\mathrm{~s}, 3 \mathrm{H}), 5.17(\mathrm{~s}, 2 \mathrm{H}), 6.38(\mathrm{~s}, 1 \mathrm{H})$, 6.89-7.33 (m, 13H), 7.64-7.66 (m, 2H); ${ }^{13} \mathrm{C} \mathrm{NMR}\left(\mathrm{CDCl}_{3}, 75 \mathrm{MHz}\right) \delta 13.22,47.36$, $108.58,112.66,125.00,125.20,126.01,126.17,126.99,127.76,128.14,128.50$, $128.92,132.37,133.56,135.70,137.97,139.38$. EI-MS (m/z, relative intensity): $355\left(\mathrm{M}^{+}, 100\right), 322$ (5), 264 (87), 249 (26), 91 (98). HRMS calcd for $\mathrm{C}_{24} \mathrm{H}_{21} \mathrm{NS}$ [M] 355.1395. Found: 355.1393.

\section{1-Allyl-5-methyl-3-phenyl-2-(phenylthio)pyrrole (7j)}

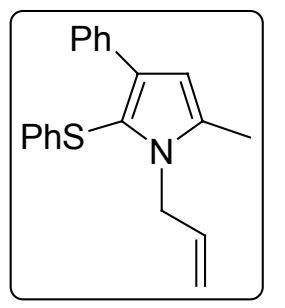

Oil; IR (film) 3060, 2915, 1582, 1510, 1477, 1439, 1404, 1336, 922, 795, 761, 739, $693 \mathrm{~cm}^{-1} ;{ }^{1} \mathrm{H}$ NMR $\left(\mathrm{CDCl}_{3}, 300 \mathrm{MHz}\right) \delta 2.28(\mathrm{~s}, 3 \mathrm{H}), 4.55-4.57(\mathrm{~m}, 2 \mathrm{H}), 4.80(\mathrm{dd}$, $J=1.2,17.1 \mathrm{~Hz}, 1 \mathrm{H}), 5.03(\mathrm{dd}, J=1.2,10.2 \mathrm{~Hz}, 1 \mathrm{H}), 5.67-5.79(\mathrm{~m}, 1 \mathrm{H}), 6.32(\mathrm{~s}$, $1 \mathrm{H}), 6.98-7.09(\mathrm{~m}, 3 \mathrm{H}), 7.16-7.21(\mathrm{~m}, 3 \mathrm{H}), 7.27-7.32(\mathrm{~m}, 2 \mathrm{H}), 7.59-7.62(\mathrm{~m}, 2 \mathrm{H})$; ${ }^{13} \mathrm{C} \mathrm{NMR}\left(\mathrm{CDCl}_{3}, 75 \mathrm{MHz}\right) \delta 12.95,46.39,108.22,111.92,116.08,124.96$, $125.16,126.09,127.73,128.09,128.91,132.23,133.44,133.85,135.74,139.56$.

EI-MS (m/z, relative intensity): 305 (M+, 100), 264 (25), 249 (11), 228 (8), 195 (17), 129 (24), 77 (13). HRMS calcd for $\mathrm{C}_{20} \mathrm{H}_{19} \mathrm{NS}$ [M] 305.1238. Found: 305.1234 .

\section{3-(4-Chlorophenyl)-5-methyl-1-phenyl-2-(phenylthio)pyrrole (2b)}

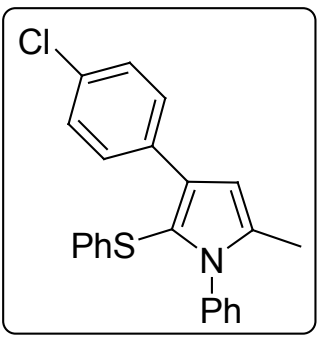

White solid; mp: $103^{\circ} \mathrm{C}$; IR (film) 3062, 1508, 1497, 832, 792, 736, $696 \mathrm{~cm}^{-1}$; ${ }^{1} \mathrm{H} \mathrm{NMR}\left(\mathrm{CDCl}_{3}, 300 \mathrm{MHz}\right) \delta 2.09(\mathrm{~s}, 3 \mathrm{H}), \quad 6.38(\mathrm{~s}, 1 \mathrm{H}), 6.84-6.87(\mathrm{~m}, 2 \mathrm{H})$, 6.99-7.14 (m, 5H), 7.25-7.30 (m, 5H), 7.60-7.63 (m, 2H); ${ }^{13} \mathrm{C} \mathrm{NMR}\left(\mathrm{CDCl}_{3}\right.$, $75 \mathrm{MHz}) \delta 13.53,108.17,114.26,125.00,125.50,128.18,128.28,128.49$, $128.53,128.69,129.00,131.25,131.97,133.97,134.35,137.75,139.24$. EI-MS (m/z, relative intensity): $375\left(\mathrm{M}^{+}, 100\right), 342$ (4), 298 (8), 263 (20), 118 
(11), 77 (26). HRMS calcd for $\mathrm{C}_{23} \mathrm{H}_{18} \mathrm{NSCl}$ [M] 375.0848. Found: 375.0849.

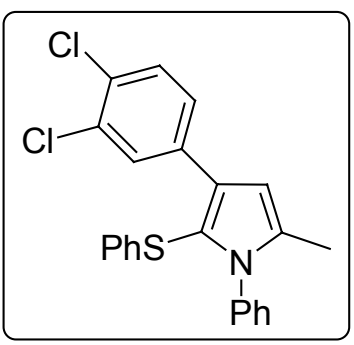

3-(3,4-Dichlorophenyl)-5-methyl-1-phenyl-2-(phenylthio) pyrrole (2c) White solid; mp: $99{ }^{\circ} \mathrm{C}$; IR (film) 3071, 1594, 1497, 767, 737, $696 \mathrm{~cm}^{-1} ;{ }^{1} \mathrm{H}$ NMR $\left(\mathrm{CDCl}_{3}, 300 \mathrm{MHz}\right) \delta 2.08(\mathrm{~s}, 3 \mathrm{H}), 6.37(\mathrm{~s}, 1 \mathrm{H}), 6.82-6.85(\mathrm{~m}, 2 \mathrm{H})$, 7.00-7.14 (m, 5H), 7.26-7.35 (m, 4H), 7.52-7.56 (m, 1H), 7.78-7.79 (m, $1 \mathrm{H}) ;{ }^{13} \mathrm{C} \mathrm{NMR}\left(\mathrm{CDCl}_{3}, 75 \mathrm{MHz}\right) \delta 13.50,108.10,114.96,125.20,125.66$, $126.99,128.30,128.47,128.60,128.76,129.00,129.47,129.87,130.01$, 132.00, 134.51, 135.66, 137.62, 138.82. EI-MS (m/z, relative intensity): $409\left(\mathrm{M}^{+}, 100\right), 376$ (4), 297 (21), 218 (18), 109 (20), 77 (46). HRMS calcd for $\mathrm{C}_{23} \mathrm{H}_{17} \mathrm{NSCl}_{2}$ [M] 409.0459. Found: 409.0458.

\section{3-(4-Methoxyphenyl)-5-methyl-1-phenyl-2-(phenylthio) pyrrole (2d)}

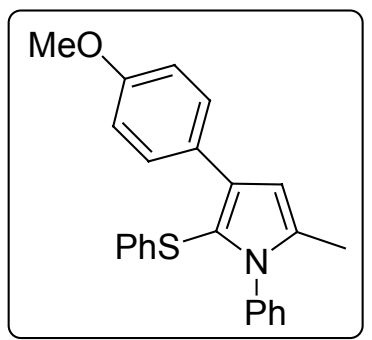

White solid; mp: $101-102{ }^{\circ} \mathrm{C}$; IR (film) 2933, 1521, 1497, 1246, 1178, 1035 , 833, 776, 739, $697 \mathrm{~cm}^{-1} ;{ }^{1} \mathrm{H}$ NMR $\left(\mathrm{CDCl}_{3}, 300 \mathrm{MHz}\right) \delta 2.09(\mathrm{~s}, 3 \mathrm{H}), 3.74(\mathrm{~s}$, $3 \mathrm{H}), 6.38(\mathrm{~s}, 1 \mathrm{H}), 6.84-6.89(\mathrm{~m}, 4 \mathrm{H}), 6.97-7.14(\mathrm{~m}, 5 \mathrm{H}), 7.24-7.29(\mathrm{~m}, 3 \mathrm{H})$, 7.58-7.62 (m, 2H); ${ }^{13} \mathrm{C}$ NMR $\left(\mathrm{CDCl}_{3}, 75 \mathrm{MHz}\right) \delta 13.54,55.10,108.18$, $113.35,113.62,124.78,125.46,127.99,128.10,128.45,128.57,128.60$, $128.87,132.36,134.02,138.00,139.80,158.21$. EI-MS $(\mathrm{m} / \mathrm{z}$, relative intensity): 371 ( $\left.\mathrm{M}^{+}, 100\right), 356$ (5), 338 (4), 294 (12), 279 (4), 118 (10), 77

(22). HRMS calcd for $\mathrm{C}_{24} \mathrm{H}_{21} \mathrm{NOS}$ [M] 371.1344. Found: 371.1346 .

\section{5-Methyl-3-(1-naphthyl)-1-phenyl-2-(phenylthio) pyrrole (2e)}

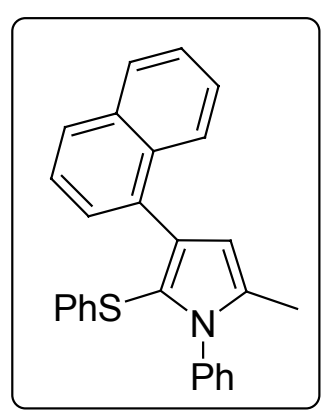

White solid; mp: $160{ }^{\circ} \mathrm{C}$; IR (film) 3059, 1497, 791, 781, 771, 740, $692 \mathrm{~cm}^{-1}$; ${ }^{1} \mathrm{H} \mathrm{NMR}\left(\mathrm{CDCl}_{3}, 300 \mathrm{MHz}\right) \delta 2.16(\mathrm{~s}, 3 \mathrm{H}), 6.39(\mathrm{~s}, 1 \mathrm{H}), 6.77-6.80(\mathrm{~m}, 2 \mathrm{H})$, 6.90-7.04 (m, 3H), 7.12-7.16 (m, 2H), 7.29-7.31 (m, 3H), 7.39-7.50 (m, 4H), 7.75-7.85 (m, 2H), 8.20-8.24 (m, 1H); ${ }^{13} \mathrm{C}$ NMR $\left(\mathrm{CDCl}_{3}, 75 \mathrm{MHz}\right) \delta 13.64$, $111.04,116.53,124.83,125.24,125.38,125.50,126.19,126.52,127.20$, $127.88,128.00,128.15,128.34,128.49,128.66,131.65,132.67,133.42$, 133.74, 133.79, 138.11, 139.47. EI-MS (m/z, relative intensity): $391\left(\mathrm{M}^{+}, 100\right)$, 358 (4), 314 (8), 299 (6), 282 (6), 77 (16). HRMS calcd for $\mathrm{C}_{27} \mathrm{H}_{21} \mathrm{NS}$ [M] 391.1395. Found: 391.1390.

\section{3-(4-Bromophenyl)-5-methyl-1-phenyl-2-(phenylthio)-1H-pyrrole (2f)}

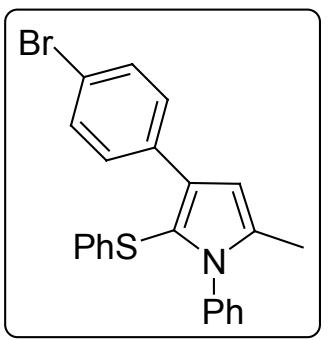

White solid; mp: $101-102{ }^{\circ} \mathrm{C}$; IR (film) 3063, 1597, 1498, 1072, 1010, 908, 830, 791, 751, 736, $695 \mathrm{~cm}^{-1}$; ${ }^{1} \mathrm{H}$ NMR $\left(\mathrm{CDCl}_{3}, 300 \mathrm{MHz}\right) \delta 2.10(\mathrm{~s}, 3 \mathrm{H})$, $6.39(\mathrm{~s}, 1 \mathrm{H}), 6.84-6.86(\mathrm{~m}, 2 \mathrm{H}), 7.00-7.15(\mathrm{~m}, 5 \mathrm{H}), 7.27-7.32(\mathrm{~m}, 3 \mathrm{H})$, 7.42-7.44 (m, 2H), 7.54-7.57 (m, 2H); ${ }^{13} \mathrm{C} \mathrm{NMR}\left(\mathrm{CDCl}_{3}, 75 \mathrm{MHz}\right) \delta 13.55$, $108.14,114.39,120.20,125.04,125.59,128.21,128.53,128.57,128.72$, $129.39,131.25,134.40,134.47,137.80,139.22$. EI-MS $(\mathrm{m} / \mathrm{z}$, relative intensity): $419\left(\mathrm{M}^{+}, 100\right), 386$ (3), 340 (6), 263 (42), 118 (20), 77 (48).

HRMS calcd for $\mathrm{C}_{23} \mathrm{H}_{18} \mathrm{NS}^{79} \mathrm{Br}[\mathrm{M}]$ 419.0343. Found: 419.0345 . 


\section{2-(Benzylthio)-5-methyl-1,3-diphenyl-pyrrole (2g)}

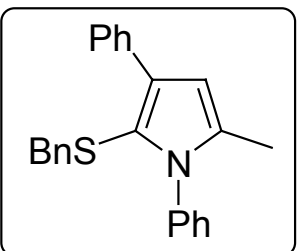

White solid; mp: $75-76{ }^{\circ} \mathrm{C}$; IR (film) 2918, 1597, 1496, 761, 750, $695 \mathrm{~cm}^{-1} ;{ }^{1} \mathrm{H}$ $\mathrm{NMR}\left(\mathrm{CDCl}_{3}, 300 \mathrm{MHz}\right) \delta 2.03(\mathrm{~s}, 3 \mathrm{H}), 3.40(\mathrm{~s}, 2 \mathrm{H}), 6.26(\mathrm{~s}, 1 \mathrm{H}), 6.75-6.78$ $(\mathrm{m}, 2 \mathrm{H}), 6.96-6.99(\mathrm{~m}, 2 \mathrm{H}), 7.06-7.13(\mathrm{~m}, 3 \mathrm{H}), 7.21-7.26(\mathrm{~m}, 2 \mathrm{H}), 7.33-7.41$ $(\mathrm{m}, 4 \mathrm{H}), 7.71-7.74(\mathrm{~m}, 2 \mathrm{H}) ;{ }^{13} \mathrm{C} \mathrm{NMR}\left(\mathrm{CDCl}_{3}, 75 \mathrm{MHz}\right) \delta 13.44,41.23,108.02$, $117.33,125.92,126.71,127.84,127.92,128.06,128.08,128.55,128.88$, $128.94,130.79,132.88,136.00,137.51,138.29$. EI-MS (m/z, relative intensity): 355 ( $\left.\mathrm{M}^{+}, 31\right), 264$ (100), 249 (11), 128 (6), 91 (9), 77 (13). HRMS calcd for $\mathrm{C}_{24} \mathrm{H}_{21} \mathrm{NS}$ [M] 355.13945. Found: 355.1397.

\section{4,5-Dimethyl-1,3-diphenyl-2-(phenylthio)pyrrole (2h)}

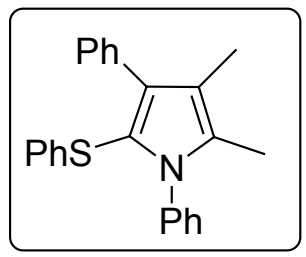

White solid; mp: 89-90 ${ }^{\circ} \mathrm{C}$; IR (film) 3060, 1597, 1497, 1367, 752, 737, 696 $\mathrm{cm}^{-1} ;{ }^{1} \mathrm{H}$ NMR $\left(\mathrm{CDCl}_{3}, 300 \mathrm{MHz}\right) \delta 2.06(\mathrm{~s}, 3 \mathrm{H}), 2.12(\mathrm{~s}, 3 \mathrm{H}), 6.81-6.84(\mathrm{~m}$, $2 \mathrm{H}), 6.96-7.12(\mathrm{~m}, 5 \mathrm{H}), 7.21-7.42(\mathrm{~m}, 8 \mathrm{H}) ;{ }^{13} \mathrm{C} \mathrm{NMR}\left(\mathrm{CDCl}_{3}, 75 \mathrm{MHz}\right) \delta 10.56$, $11.52,113.93,114.91,124.69,125.75,126.28,127.80,127.83,128.41,128.43$, $128.67,129.95,130.83,133.30,135.43,138.30,140.18$. EI-MS (m/z, relative intensity): 355 ( $\left.\mathrm{M}^{+}, 100\right), 322$ (23), 278 (19), 263 (11), 118 (12), 77 (35). HRMS calcd for $\mathrm{C}_{24} \mathrm{H}_{21} \mathrm{NS}$ [M] 355.1395. Found: 355.1390.

\section{3,5-Dimethyl-1-phenyl-2-(phenylthio)pyrrole (2j)}

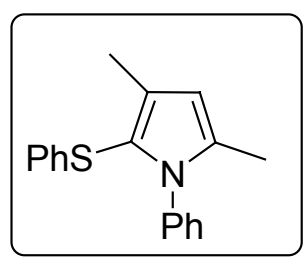

White solid; mp: 122-123 ${ }^{\circ} \mathrm{C}$; IR (film) 3067, 2916, 1598, 1582, 1499, 1477, 1354, $1023 \mathrm{~cm}^{-1}$; ${ }^{1} \mathrm{H}$ NMR $\left(\mathrm{CDCl}_{3}, 300 \mathrm{MHz}\right) \delta 2.08(\mathrm{~s}, 3 \mathrm{H}), 2.19$ (s, 3H), 6.07 $(\mathrm{s}, 1 \mathrm{H}), 6.85-6.88(\mathrm{~m}, 2 \mathrm{H}), 7.01-7.07(\mathrm{~m}, 3 \mathrm{H}), 7.12-7.18(\mathrm{~m}, 2 \mathrm{H}), 7.29-7.32(\mathrm{~m}$, $3 \mathrm{H}) ;{ }^{13} \mathrm{C} \mathrm{NMR}\left(\mathrm{CDCl}_{3}, 75 \mathrm{MHz}\right) \delta 12.02,13.52,109.32,114.82,124.67,125.27$, $127.77,128.44,128.49,128.60,133.34,138.36,139.78$. EI-MS (m/z, relative intensity): 279 ( $\left.\mathrm{M}^{+}, 100\right), 264$ (5), 246 (28), 202 (39), 187 (15), 77 (68). HRMS calcd for $\mathrm{C}_{18} \mathrm{H}_{17} \mathrm{NS}$ [M] 279.1082. Found: 279.1092.

\section{2-(3-Methoxyphenyl)-2-(benzylthio)penta-3,4-dienal (4)}

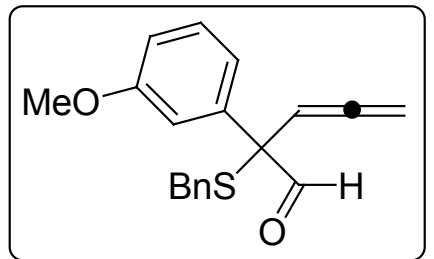

Oil; IR (film) 2834, 1709, 1599, 1582, 1493, 1260, 1051, 854, 777, 696 $\mathrm{cm}^{-1} ;{ }^{1} \mathrm{H}$ NMR $\left(\mathrm{CDCl}_{3}, 300 \mathrm{MHz}\right) \delta 3.57(\mathrm{~d}, J=12.0 \mathrm{~Hz}, 1 \mathrm{H}), 3.68(\mathrm{~d}$, $J=12.0 \mathrm{~Hz}, 1 \mathrm{H}), 3.80(\mathrm{~s}, 3 \mathrm{H}), 4.87-4.98(\mathrm{~m}, 2 \mathrm{H}), 5.73(\mathrm{t}, \quad J=6.6 \mathrm{~Hz}$, $1 \mathrm{H}), 6.85-6.89(\mathrm{~m}, 1 \mathrm{H}), 7.05-7.08(\mathrm{~m}, 2 \mathrm{H}), 7.22-7.34(\mathrm{~m}, 6 \mathrm{H}), 9.38(\mathrm{~s}$, $1 \mathrm{H}) ;{ }^{13} \mathrm{C} \mathrm{NMR}\left(\mathrm{CDCl}_{3}, 75 \mathrm{MHz}\right) \delta 34.60,55.25,63.04,78.88,90.56$, $113.72,113.99,120.33,127.38,128.58,129.23,129.74,136.04,137.48$, 159.79, 188.40, 209.21. EI-MS (m/z, relative intensity): 310 (M+ , 8), 281 (6), 219 (23), 187 (23), 115 (17), 91 (100). HRMS calcd for $\mathrm{C}_{19} \mathrm{H}_{18} \mathrm{O}_{2} \mathrm{~S}$ [M] 310.1028. Found: 310.1019. 
2-(Benzylthio)-3-(3-methoxyphenyl)-5-methyl-1-phenyl-pyrrole (5)

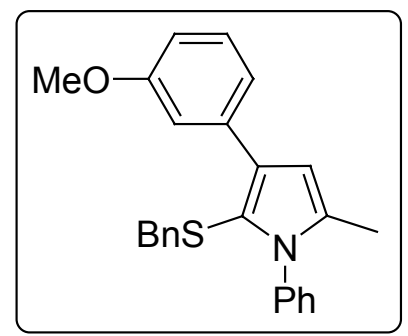

Oil; IR (film) 2938, 1598, 1497, 775, 739, $697 \mathrm{~cm}^{-1} ;{ }^{1} \mathrm{H}$ NMR $\left(\mathrm{CDCl}_{3}\right.$, $300 \mathrm{MHz}) \delta 2.03(\mathrm{~s}, 3 \mathrm{H}), 3.43(\mathrm{~s}, 2 \mathrm{H}), 3.84(\mathrm{~s}, 3 \mathrm{H}), 6.27(\mathrm{~s}, 1 \mathrm{H})$, 6.76-6.83 (m, 3H), 6.94-6.98 (m, 2H), 7.08-7.14 (m, 3H), 7.31-7.41 (m, $6 \mathrm{H}) ;{ }^{13} \mathrm{C}$ NMR $\left(\mathrm{CDCl}_{3}, 75 \mathrm{MHz}\right) \delta 13.44,41.22,55.18,108.03,111.75$, $113.18,117.51,120.46,126.71,127.87,128.08,128.55,128.88,128.93$, $128.99,130.42,132.86,137.38,137.54,138.24,159.38$. EI-MS (m/z, relative intensity): $385\left(\mathrm{M}^{+}, 15\right), 294$ (50), 157 (44), 128 (54), 91 (100), 77 (34). HRMS calcd for $\mathrm{C}_{25} \mathrm{H}_{23} \mathrm{NOS}$ [M] 385.1500. Found: 385.1498 . 